Supporting information for

\title{
Directing Selectivity of Electrochemical Carbon Dioxide Reduction Using Plasmonics
}

Erin B. Creel, ${ }^{1,2,3}$ Elizabeth R. Corson, ${ }^{1,4}$ Johanna Eichhorn, ${ }^{1}$ Robert Kostecki, ${ }^{1,5}$ Jeffrey J. Urban, ${ }^{1,3}$ Bryan D. McCloskey ${ }^{1,4,5 *}$

${ }^{1}$ Joint Center for Artificial Photosynthesis, Lawrence Berkeley National Laboratory, Berkeley, California 94720, USA

${ }^{2}$ Department of Chemistry, University of California, Berkeley, California 94720, USA

${ }^{3}$ The Molecular Foundry, Lawrence Berkeley National Laboratory, Berkeley, California 94720, USA

${ }^{4}$ Department of Chemical and Biomolecular Engineering, University of California, Berkeley, California 94720, USA

${ }^{5}$ Energy Storage and Distributed Resources Division, Lawrence Berkeley National Laboratory, Berkeley, California 94720, USA 


\section{Supporting Information Methods}

\section{Synthesis of silver cathodes}

3 in. $\times 1$ in. $\times 1.2 \mathrm{~mm}$ glass microscope slides were cleaned by sonication in acetone for 10 minutes followed by oxygen plasma cleaning for $4 \mathrm{~min}$. The catalyst was fabricated by e-beam evaporation of $5 \mathrm{~nm}$ of titanium (99.999\%) as an adhesion layer on the clean glass slide and $200 \mathrm{~nm}$ of silver $(99.97 \%)$ over the titanium. Silver cathodes were stored in a vacuum chamber after fabrication until use. Except where noted, all silver cathodes were electrochemically conditioned at $-1.1 \mathrm{~V}$ versus the reversible hydrogen electrode $\left(\mathrm{V}_{\mathrm{RHE}}\right)$ for $45 \mathrm{~min}$ in carbon-dioxide- $\left(\mathrm{CO}_{2}\right)$ saturated aqueous $1 \mathrm{M}$ potassium bicarbonate $\left(\mathrm{KHCO}_{3}\right)$ before use.

\section{X-ray photoelectron spectroscopy}

XPS spectra were collected using a Thermo Scientific K-Alpha XPS System with a monochromated aluminum $\mathrm{K} \alpha$ source. A pass energy of $100 \mathrm{eV}$ was used for survey scans and $20 \mathrm{eV}$ for high resolution scans with an energy resolution of $0.1 \mathrm{eV}$. Thin film surfaces were cleaned using an argon cluster gun (6000 eV, 150 atoms per cluster) for $60 \mathrm{~s}$ before all XPS measurements. XPS spectra show the characteristic silver $3 \mathrm{~d}_{3 / 2}$ and $3 \mathrm{~d}_{5 / 2}$ peaks but do not show any sign of the underlying titanium glass adhesion layer after electrochemical conditioning.

\section{Cathode surface imaging}

Scanning electron microscopy (SEM) images were acquired using a Thermo Scientific Quanta FEG 250 SEM and a $10 \mathrm{kV}$ accelerating voltage.

Atomic force microscopy (AFM) measurements were acquired with a Bruker Dimension Icon commercial AFM system using the PeakForce Quantitative Nanoscale Mechanical tapping mode under ambient conditions. For all topography measurements uncoated silicon ( $\mathrm{Si}$ ) probes (SCANASYST-AIR) with spring constants of $0.4 \mathrm{~N} \mathrm{~m}^{-1}$ were used. Grain size analysis was performed using the watershed algorithm in the Gwyddion software package. The grain location was determined with 100 steps, a drop size of $0.10 \%$, and a threshold of $150 \mathrm{~nm}^{2}$. Segmentation was determined with 20 steps and a drop size of $15.00 \%$. No additional preparation for AFM was performed on the as-deposited silver electrodes, but electrodes were rinsed profusely in ultrapure water (Millipore, $18 \mathrm{M} \Omega$ ) and dried with a nitrogen stream after electrochemical conditioning in preparation for AFM measurements.

\section{Absorption measurements}

UV-visible total reflection, R, measurements of dry silver electrodes were collected on a Shimadzu SolidSpec-3700 UV-vis-NIR spectrophotometer equipped with an integrating sphere after various electrolysis times in a beaker with $\mathrm{CO}_{2}$-saturated aqueous $1 \mathrm{M} \mathrm{KHCO}_{3}$ with a platinum wire counter electrode and a Basi MF-2052 silver/silver chloride $(\mathrm{Ag} / \mathrm{AgCl})$ reference electrode. Spectra were referenced to a NIST-calibrated mirror, and the calibration 
was used to find the absolute reflection values. Absorption values reported here are $100 \%-$ $\% \mathrm{R}$.

\section{Photoelectrochemical measurements}

The electrolyte used in this study was $\mathrm{CO}_{2}$-saturated aqueous $1 \mathrm{M} \mathrm{KHCO}_{3}(\mathrm{pH} 7.75)$ made by saturating $0.5 \mathrm{M}$ potassium carbonate $\left(\mathrm{K}_{2} \mathrm{CO}_{3}\right)$ (Alfa Aesar, $99.997 \%$ metals basis) prepared with ultrapure water (Millipore, $18 \mathrm{M} \Omega$ ). The electrolyte was sparged with $\mathrm{CO}_{2}$ for 10 minutes prior to electrolysis start. 85\%-IR-corrected measurements were performed with a Biologic SP-300 potentiostat in a gas-tight polyether ether ketone (PEEK) electrochemical compression cell ${ }^{1}$ with Ace Seal 2-015 Kalrez 6375 o-rings exposing $1 \mathrm{~cm}^{2}$ geometric surface area of the cathode and anode. Impedance spectroscopy from 100 to $1 \mathrm{kHz}$ with $10 \mathrm{mV}$ amplitude at open circuit voltage was used to calculate the IR correction for each measurement. Typical resistances measured between the working and reference electrodes in our cell fell between 5 and $10 \Omega$. A temperature control system comprising a Ferrotec 72008/131/150B peltier cooler mounted flush with the backside of the cathode, CPU heat sink with fan, and proportional-integral-derivative (PID) controller held the catholyte at a constant $22.0 \pm 0.1^{\circ} \mathrm{C}$. A Selemion AMF anion exchange membrane separated the catholyte and anolyte. $\mathrm{CO}_{2}$ was continuously bubbled at $5 \mathrm{sccm}$ through a glass frit at the bottom of the cell unless otherwise noted. Platinum foil was used as the water oxidation anode, and no platinum dissolution or deposition was observed on the silver cathode, as our product Faradaic efficiencies matched those in a previous study. ${ }^{2}$ An Innovative Instruments, Inc., $\mathrm{LF}-1$ leak-free $\mathrm{Ag} / \mathrm{AgCl}$ electrode was used as the potential reference, but all potentials were converted to and reported versus the reversible hydrogen electrode. Cyclic voltammograms that were performed in Ar- or $\mathrm{CO}_{2}$-saturated $0.5 \mathrm{M} \mathrm{K}_{2} \mathrm{CO}_{3}$ or $0.5 \mathrm{M}$ sodium perchlorate $\left(\mathrm{NaClO}_{4}\right)$ at a sweep rate of $100 \mathrm{mV} \mathrm{s}^{-1}$. The cathodic sweep of the 10 th cycle, representative of any cycle, is plotted in Figures 4 and S4.

The cathode was illuminated from the front using a LCS-0365-48-22 Mightex Systems 365 $\mathrm{nm}$ ultra-high-power LED. While the output power of the LED was $2.5 \mathrm{~W}$, only $170 \mathrm{~mW}$ reached the surface of the cathode due to the size of the cell window, absorption of the electrolyte, and scattering by the $\mathrm{CO}_{2}$ bubbles. The light intensity incident on the cathode was determined by measuring the transmission of light through the photoelectrochemical cell with electrolyte, $\mathrm{CO}_{2}$ bubbles, and a glass slide in place of the thin film silver cathode then corrected for the $91.1 \%$ transmission of $365 \mathrm{~nm}$ light through the glass slide. The electrolyte was saturated, but the the $\mathrm{CO}_{2}$ bubbling was turned off for the example choppedlight chronoamperometry plot (Figure 6a) to minimize noise in the signal. The output light intensity for that measurement only was decreased to maintain an incident light intensity of $170 \mathrm{~mW} \mathrm{~cm}{ }^{-2}$. Wavelength-dependent measurements were performed at equal incident photon flux $\left(3.2 \times 10^{17}\right.$ photons $\left.\mathrm{cm}^{-2} \mathrm{~s}^{-1}\right)$ to the $365 \mathrm{~nm}$ LED using Mightex Systems LCS-0405-50-22, LCS-0470-50-22, and LCS-0525-60-22 LEDs at 405, 470, and $525 \mathrm{~nm}$, respectively. The emission spectra of the LEDs provided by the manufacturer is included as Figure S7. The output and incident power were measured with a Coherent PowerMax PM10 power meter connected to a Coherent LabMax-TOP power meter console. Photocurrent was extracted from $3 \mathrm{~Hz}$ chopped-light chronoamperometry measurements using a Stanford Research Systems SR850 lock-in amplifier and a ThorLabs SHB1 shutter system. 


\section{$\mathrm{CO}_{2}$ reduction product measurements}

Gaseous products of the electrochemical reactions were analyzed by an in-line SRI Instruments Multiple Gas Analyzer \#5 gas chromatograph (GC) equipped with a $12 \mathrm{ft}$ HayeSep D (divinylbenzene) column, thermal conductivity detector (TCD), flame ionization detector (FID) with methanizer, and argon carrier gas. The detection limit of the GC is $1 \mathrm{ppm}$. For a single product analysis run, a constant potential was applied for $64 \mathrm{~min}$ with GC injections at $3,15,27,39,51$, and 63 minutes. Gas in the headspace and gas line to the GC does not eqilibrate by the three minute injection, so the average concentration from only the last five cycles was used in calculations of product generation. The concentration of the gas in each injection was computed from calibration curves with at least three points for each gas type. The partial current density, $I_{x}$, for each gaseous product, $x$, in each GC injection is given by

$$
I_{x}=\frac{\mathrm{F} c_{x} n f}{m a}
$$

where $\mathrm{F}$ is Faraday's constant (96485 $\mathrm{C} \mathrm{mol}^{-1}$ of electrons), $c_{x}$ is the concentration of product $x$ as a molecular fraction of the total injected gas, $n$ is the number of electrons needed to produce product $x$ ( 2 mol of electrons per mol of hydrogen or carbon monoxide), $f$ is the flow rate of $\mathrm{CO}_{2}$ into the electrochemical cell $\left(5 \mathrm{~cm}^{3} \mathrm{~min}^{-1}\right.$ or $8.333 \times 10^{-5} \mathrm{~L} \mathrm{~s}^{-1}$ in this study), $m$ is the the molar volume of $\mathrm{CO}_{2}$ at $21{ }^{\circ} \mathrm{C}\left(24.01 \mathrm{~L} \mathrm{~mol}^{-1}\right.$ from the National Institute of Standards and Technology (NIST) WebBook), and $a$ is the geometric surface area of the cathode $\left(1 \mathrm{~cm}^{2}\right.$ in this study). We assume that the evolved gases have the similar molar volumes as $\mathrm{CO}_{2}$. The faradaic efficiency, FE, of a product is given by

$$
\mathrm{FE}=\frac{I_{x}}{I}
$$

where $I$ is the average total current density measured by the potentiostat over the five minutes before the GC injection. The average values for current density and faradaic efficiency over the last five injections are reported here. Liquid products in the catholyte and anolyte were quantified after the electrolysis was complete by ${ }^{1} \mathrm{H}$ NMR spectroscopy on a Bruker Avance III $500 \mathrm{MHz}$ magnet using phenol and dimethylsulfoxide (DMSO) as internal standards and a water suppression method with $60 \mathrm{~s}$ between pulses to allow for complete proton relaxation. ${ }^{1}$ The area of the DMSO singlet peak at $2.6 \mathrm{ppm}$ relative to tetramethylsilane (TMS) was used to determine the methanol concentration in the sample using the methanol singlet at 3.23 ppm relative to TMS. The detection limit of the NMR is $1 \mathrm{ppb}$.

\section{Electrochemical surface area measurements}

Electrochemical surface area (ECSA) of electrochemically conditioned electrodes was determined relative to the as-e-beam-evaporated electrode by taking the ratio of their double layer capacitances as measured by the cyclic linear potential sweep method. ${ }^{3}$ First, the non-Faradaic charging current in cyclic voltammograms $(\mathrm{CVs})$ was measured at a series of increasingly rapid scan rates (Figure S1a). A Savitzky-Golay filter was used to smooth the CV data in Figure S1a, but the raw data was used in the determination of the charging current. The charging current was taken as half of the difference between the average current between 0.544 and $0.549 \mathrm{~V}_{\mathrm{RHE}}$ in the anodic direction and the average current between 
0.509 and $0.514 \mathrm{~V}_{\mathrm{RHE}}$ in the cathodic direction on the third CV cycle. The capacitance is the y-intercept of the best-fit line of the charging current versus scan rate (Figure S1b). The capacitance was measured for the same electrode after various electrolysis times. Each measured capacitance was normalized to the capacitance of the as-deposited electrode for the relative ECSA (Figure 1d and Figure S1c).

\section{References}

(1) Corson, E. R.; Creel, E. B.; Kim, Y.; Urban, J. J.; Kostecki, R.; McCloskey, B. D. A temperature-controlled photoelectrochemical cell for quantitative product analysis. Rev. Sci. Instrum. 2018, 89, 055112.

(2) Hatsukade, T.; Kuhl, K. P.; Cave, E. R.; Abram, D. N.; Jaramillo, T. F. Insights into the electrocatalytic reduction of $\mathrm{CO}_{2}$ on metallic silver surfaces. Phys. Chem. Chem. Phys. 2014, 16, 13814-13819.

(3) Bard, A. J.; Faulkner, L. R. Electrochemical Methods: Fundamentals and Applications, 2nd ed.; John Wiley \& Sons, Inc.: New York, 2001; pp 14-18. 


\section{Supporting information figures}

a

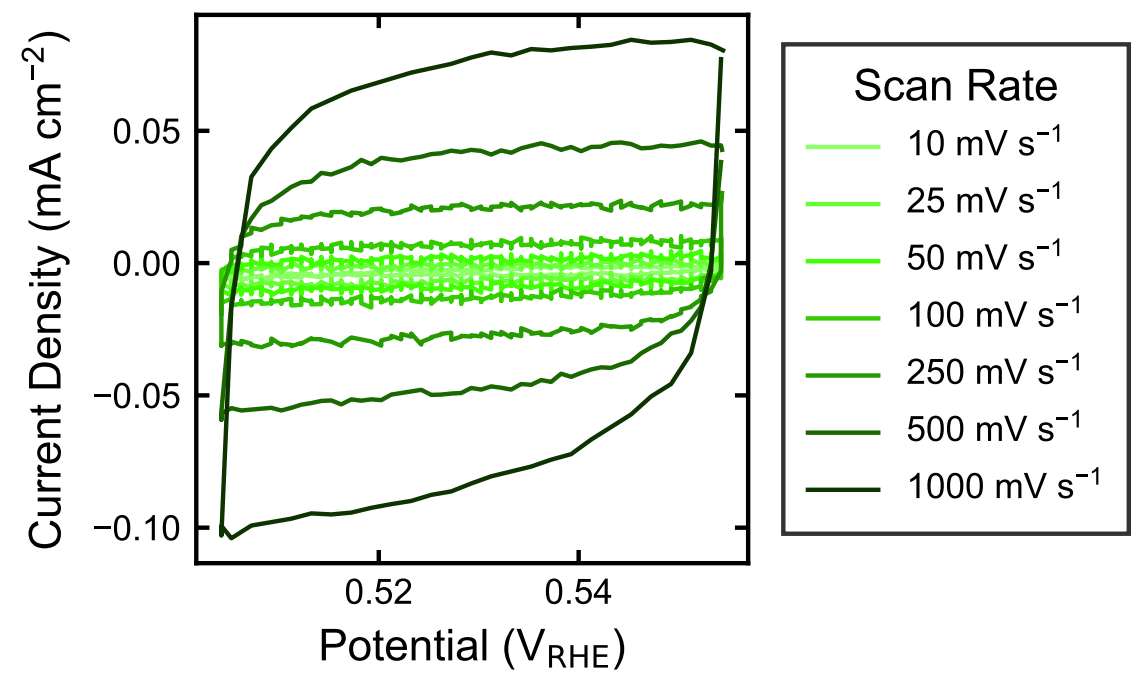

b

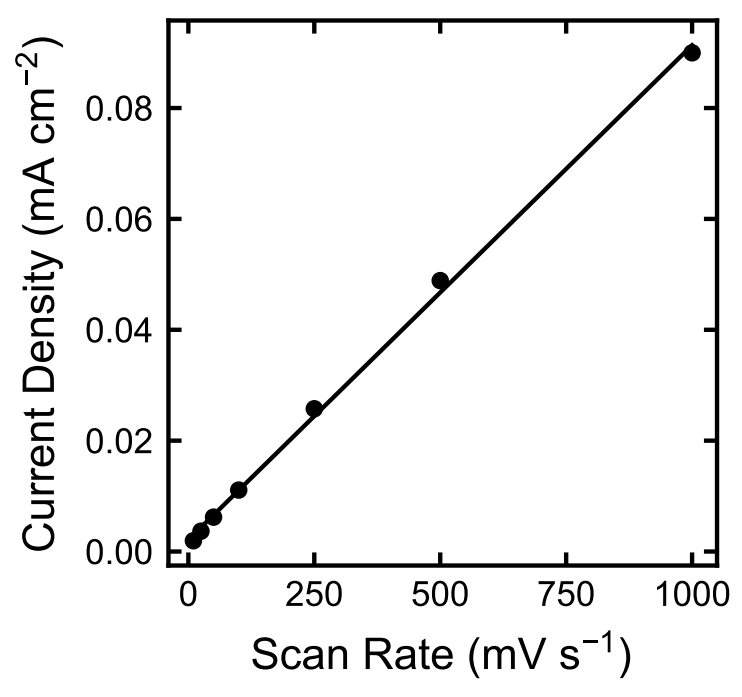

C

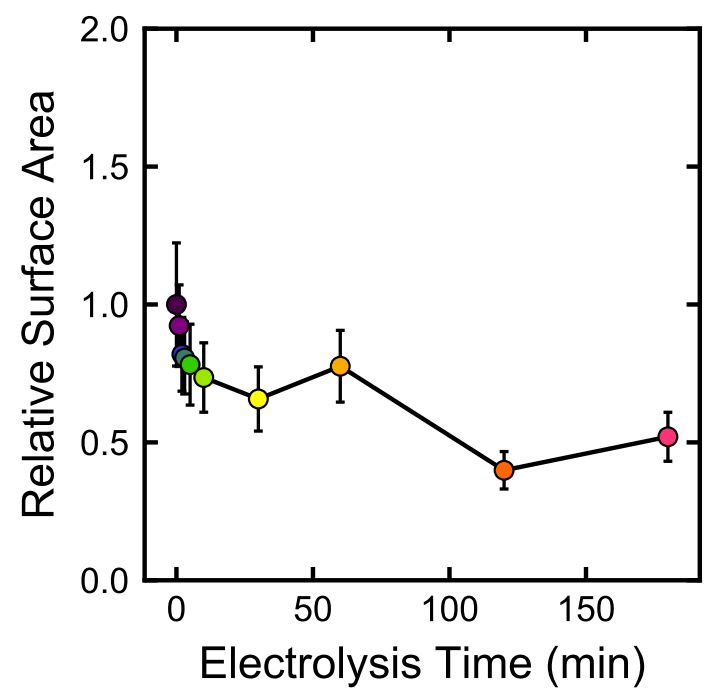

Figure S1. Relative electrochemical surface area (ECSA) measurement by capacitive cycling measurements on a silver cathode. (a) Capacitive cycles over a $50 \mathrm{mV}$ non-Faradaic region at a series of rapid scan rates after 0 minutes of electrolysis. Only the third cycle for each scan rate is shown here. (b) Capacitive current for a series of scan rates after 0 minutes of electrolysis where the y-intercept of the best-fit line gives the capacitance. (c) Capacitance relative to the unconditioned (0 minutes of electrolysis) electrode capacitance gives the relative ECSA as a function of electrolysis time. Error bars represent the standard deviation of experiments performed in triplicate on a single electrode. 
a

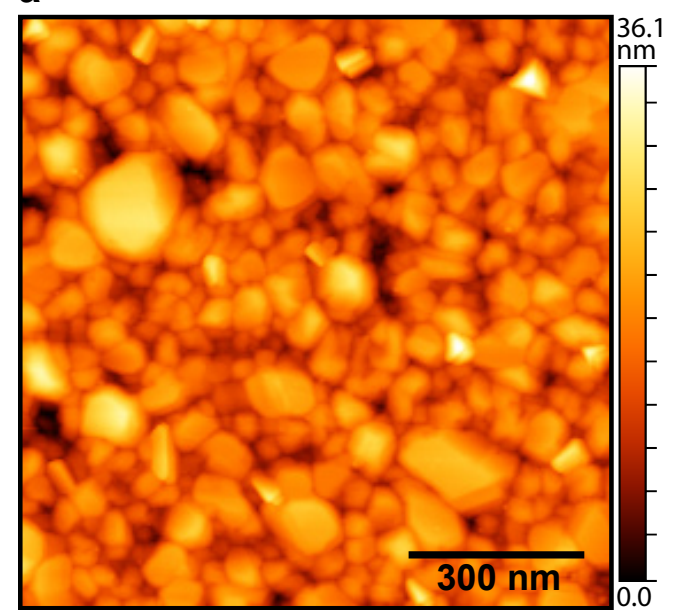

C

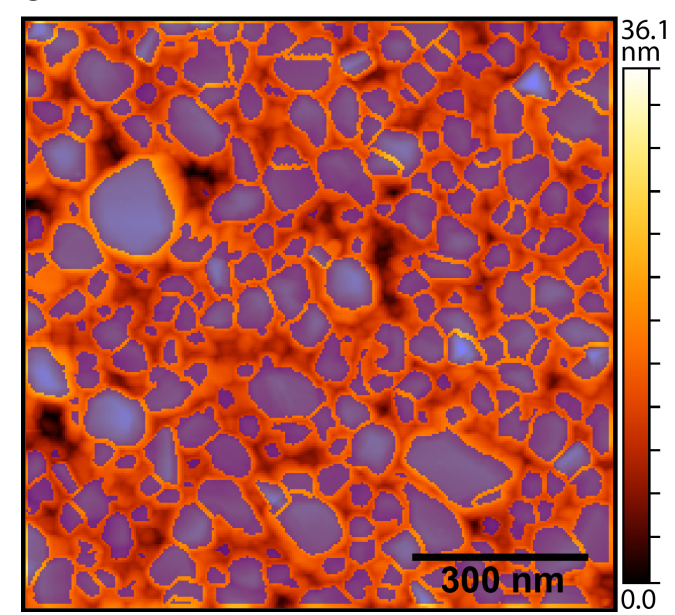

b
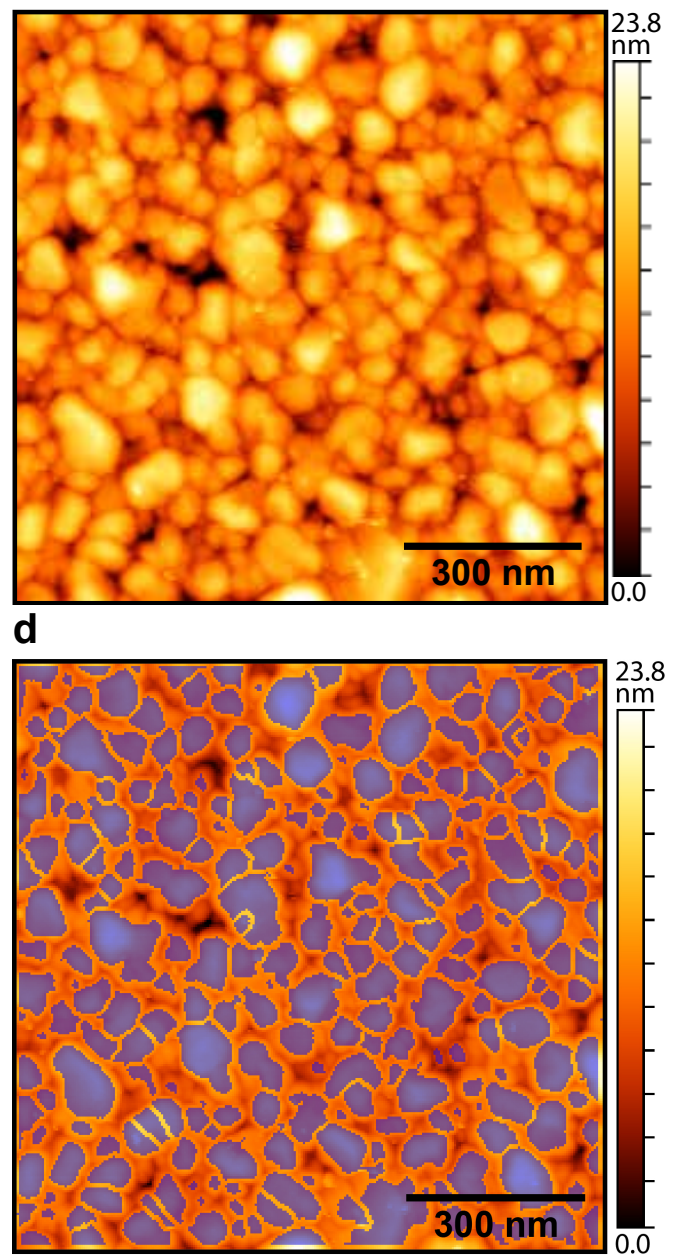

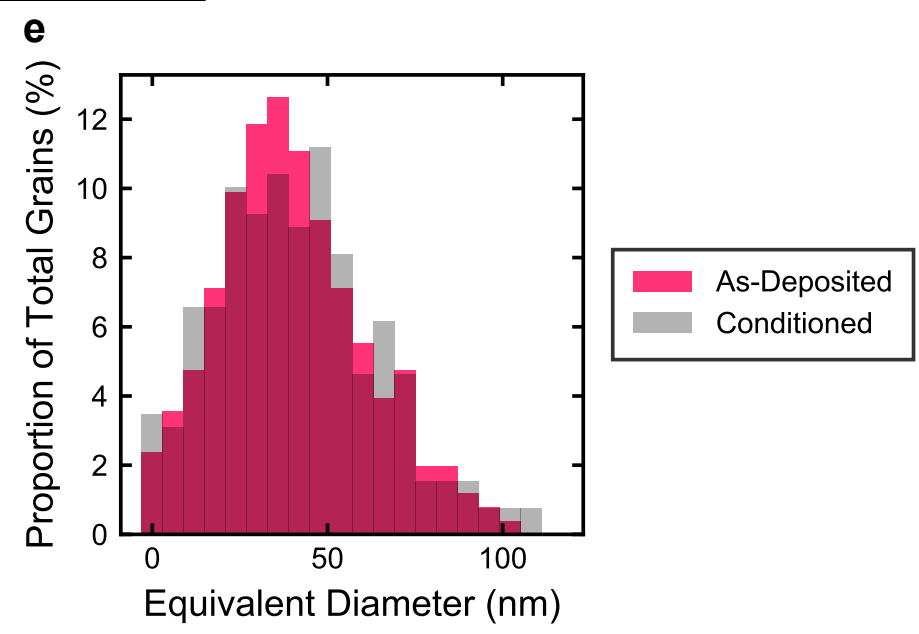

Figure S2. Atomic force microscopy (AFM) topographic images of the thin film silver cathode (a) as-deposited and (b) after biasing at $-1.1 \mathrm{~V}_{\mathrm{RHE}}$ for 45 minutes. Grains detected by the watershed algorithm (purple) in the AFM image of the cathode (c) as-deposited and (d) after biasing at $-1.1 \mathrm{~V}_{\mathrm{RHE}}$ for 45 minutes. (e) Comparison of the grain size distribution on the cathode in terms of equivalent disc diameter, the diameter of the disc with the same projected area as the grain, "As-Deposited" and after biasing at -1.1 $\mathrm{V}_{\mathrm{RHE}}$ for 45 minutes ("Conditioned"). 
a

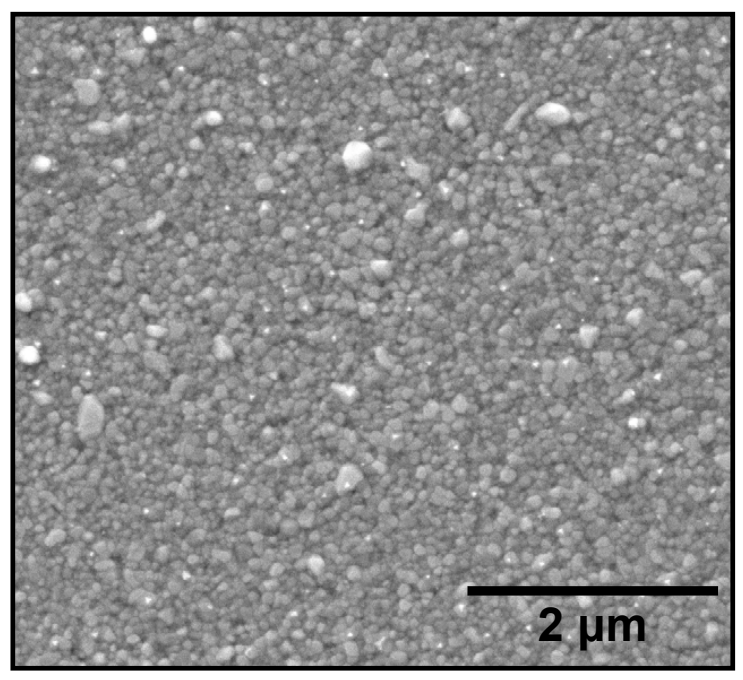

b

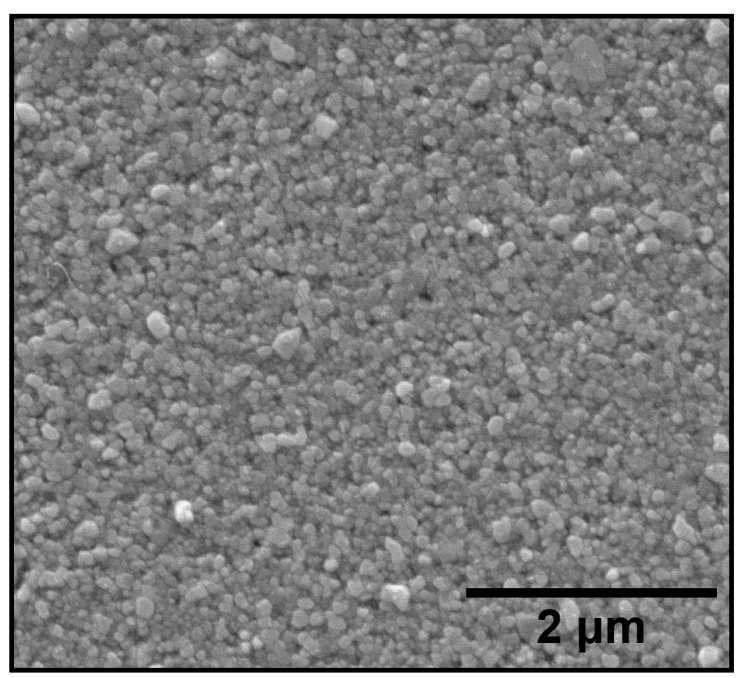

Figure S3. Scanning electron micrographs of the thin film silver cathode (a) as-deposited and (b) after biasing at $-1.1 \mathrm{~V}_{\mathrm{RHE}}$ for 45 minutes.
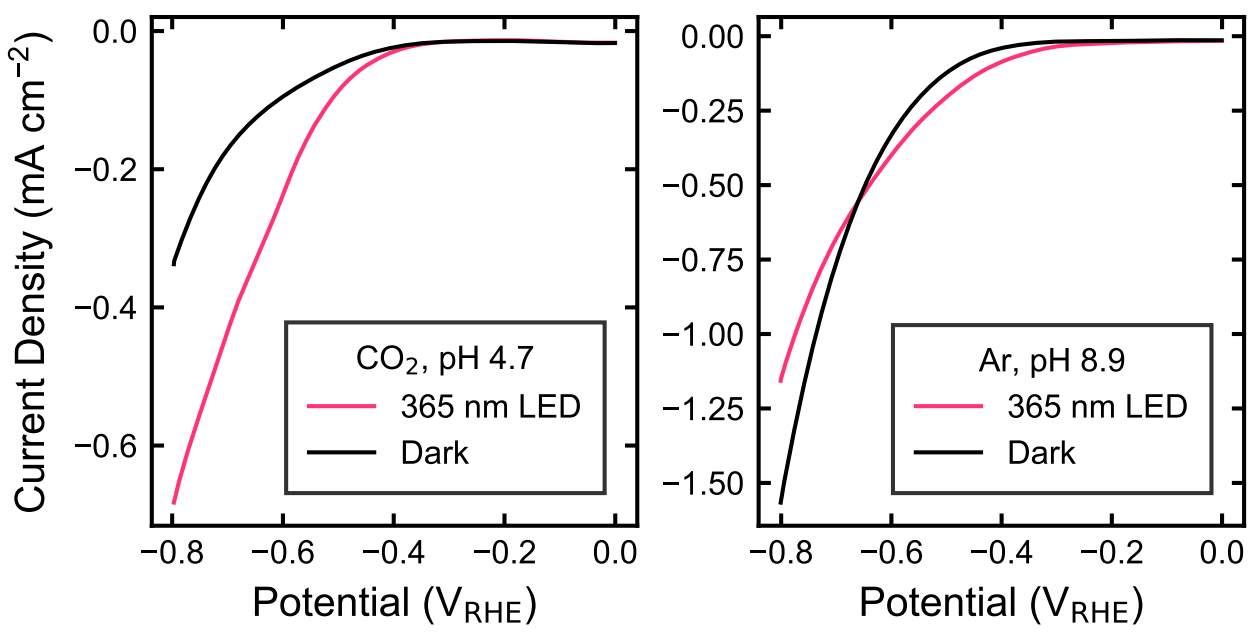

Figure S4. Cathodic voltage sweep at $100 \mathrm{mV} \mathrm{s}^{-1}$ over an illuminated and dark silver electrodes in (a) $\mathrm{CO}_{2^{-}}$and (b) Ar-saturated $0.5 \mathrm{M} \mathrm{NaClO}_{4}$ under $365 \mathrm{~nm}$ LED light at $170 \mathrm{~mW} \mathrm{~cm}^{-2}$. The voltage ranges were selected to ensure no silver oxidation occurred at the anodic limit and to include all potentials where a photoeffect is observed for $\mathrm{CO}$ and hydrogen in $\mathrm{CO}_{2}$-saturated $\mathrm{K}_{2} \mathrm{CO}_{3}$ at the cathodic limit. 
a

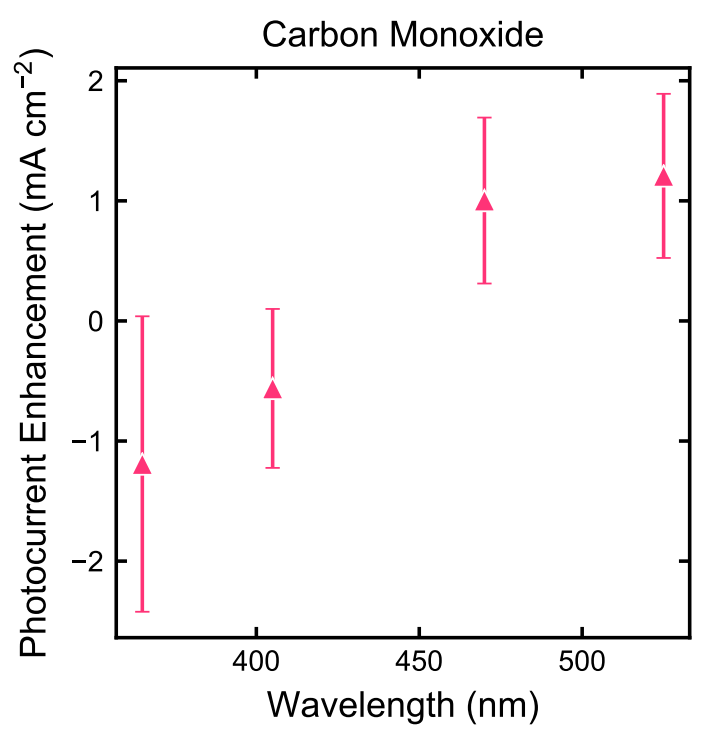

C

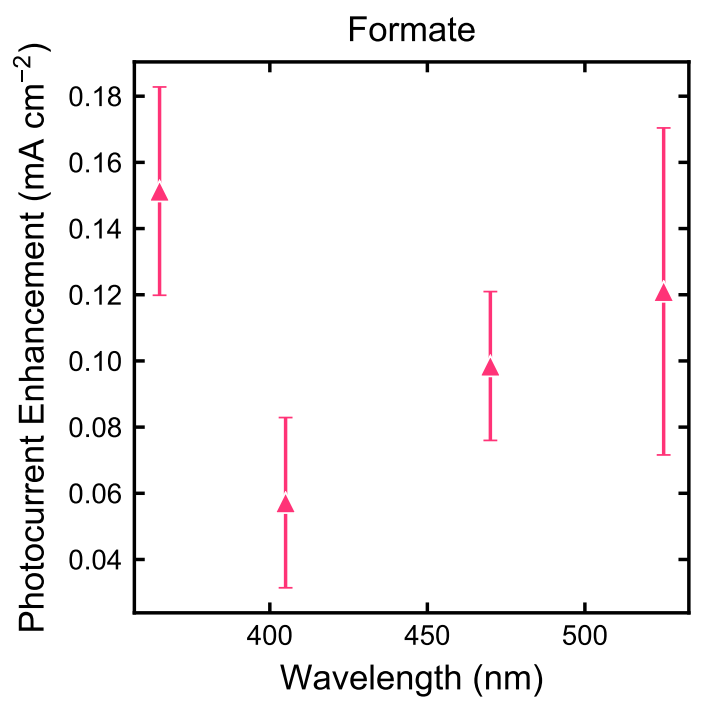

b

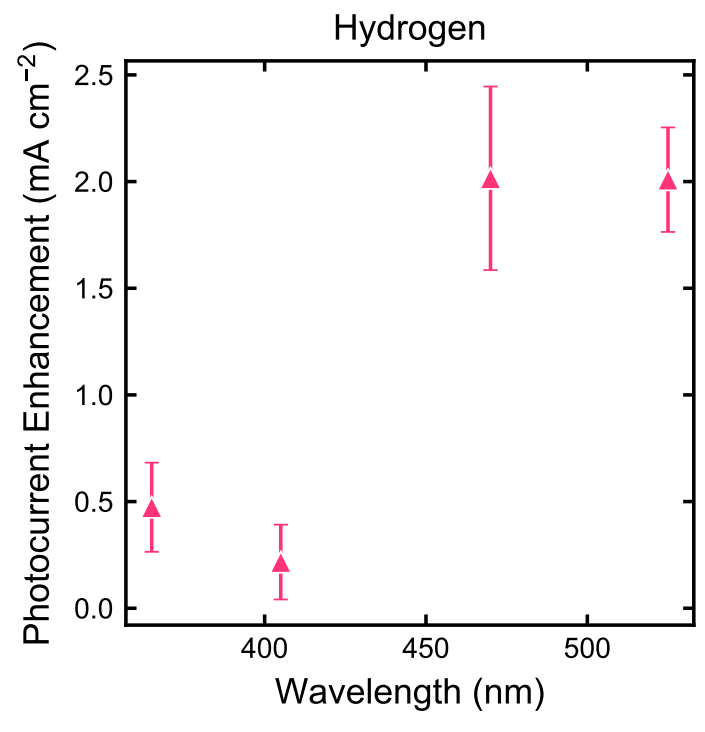

d

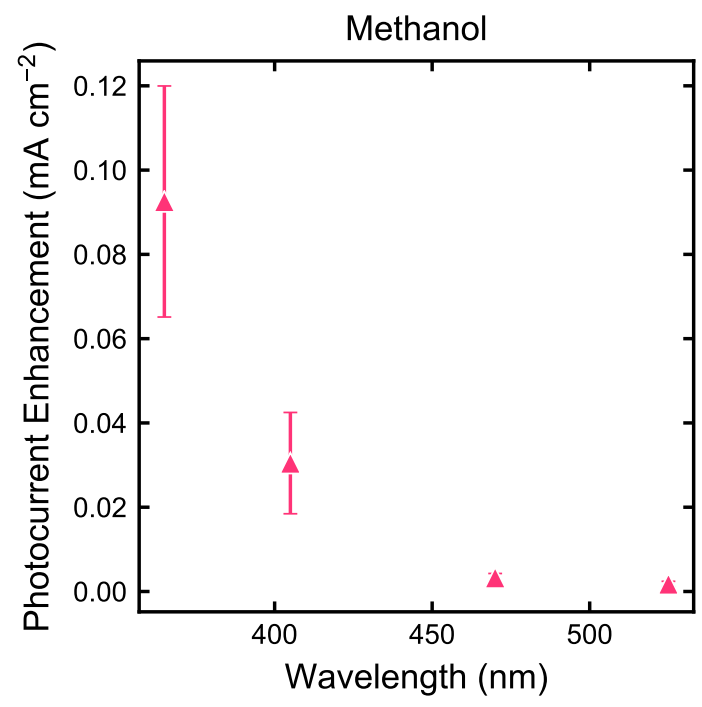

Figure S5. Difference between the partial current density of each product under $3.2 \times 10^{17}$ photons $\mathrm{cm}^{-2} \mathrm{~s}^{-1}$ illumination of various wavlengths and in dark conditions at $-1.1 \mathrm{~V}_{\text {RHE }}$. (a) carbon monoxide, (b) hydrogen, (c) formate, and (d) methanol 


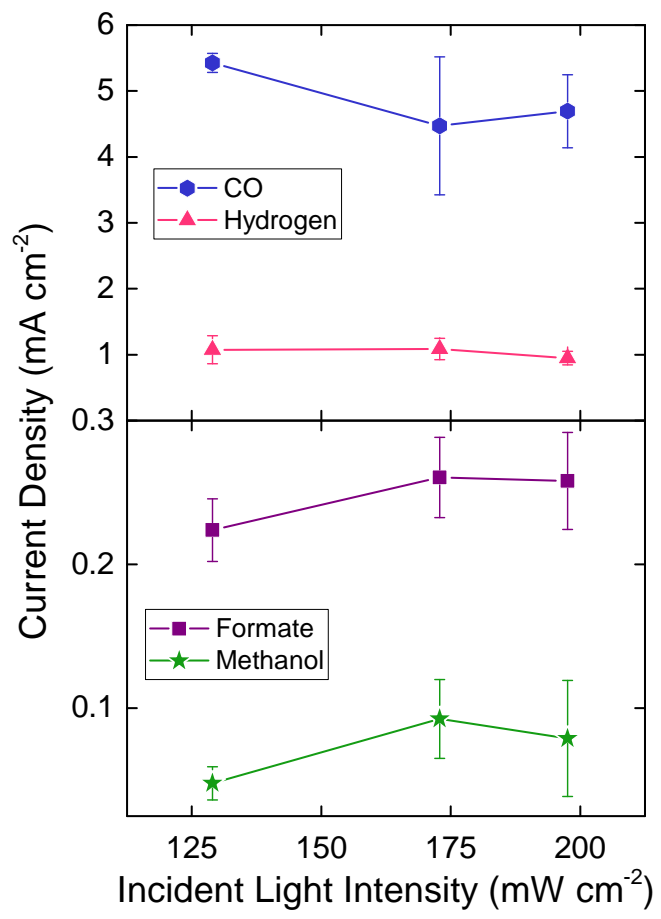

Figure S6. Partial current density for each product at $-1.1 \mathrm{~V}_{\mathrm{RHE}}$ under $365 \mathrm{~nm}$ LED illumination of various incident light intensities. Error bars represent the standard deviation of experiments performed in triplicate. 
a

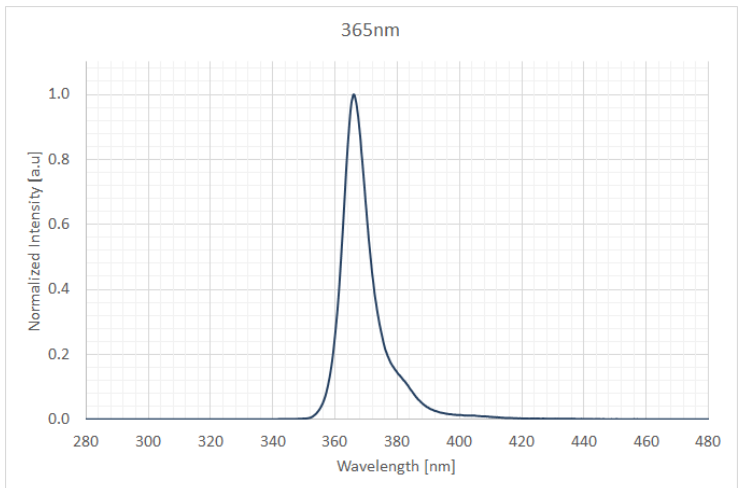

C

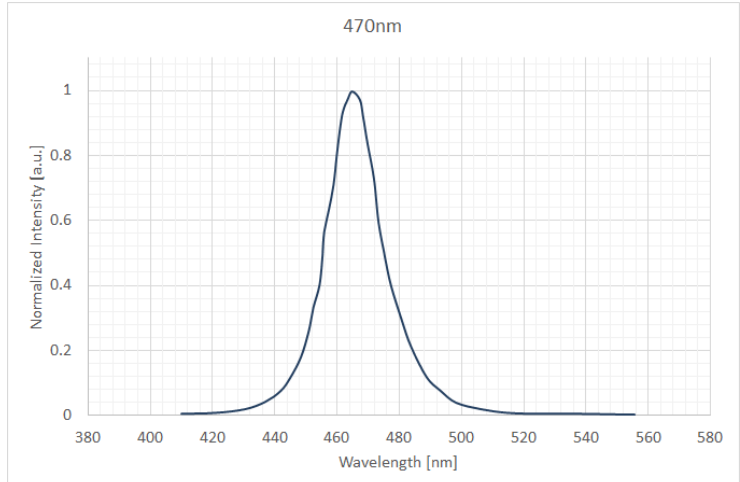

b

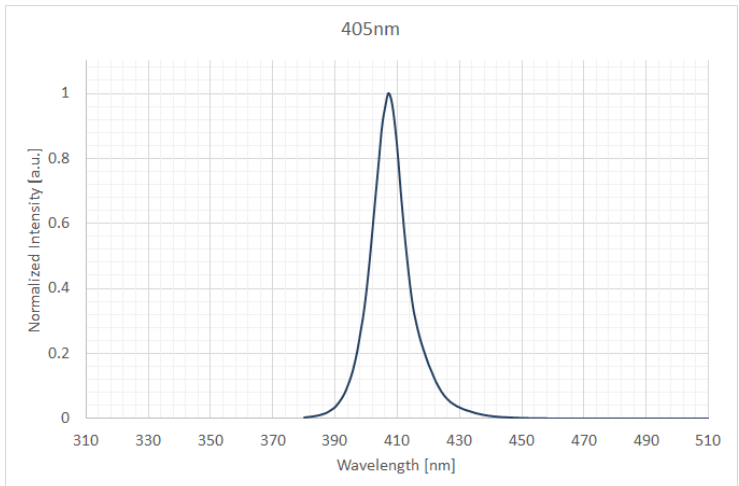

d

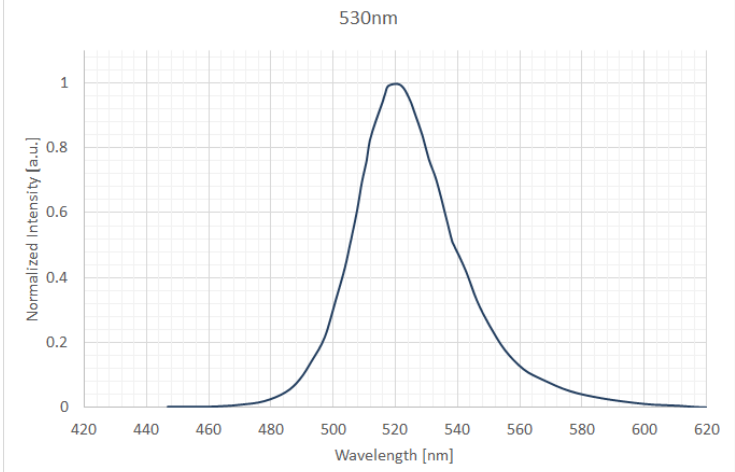

Figure S7. Emission spectra of the ultra-high-power Mightex Systems LEDs used in this study provided by Mightex. (a) $365 \mathrm{~nm}$ LED (LCS-0365-48)-22, (b) $405 \mathrm{~nm}$ LED (LCS0405-50-22), (c) $470 \mathrm{~nm}$ LED (LCS-0470-50-22), and (d) $525 \mathrm{~nm}$ LED (LCS-0525-60-22) 